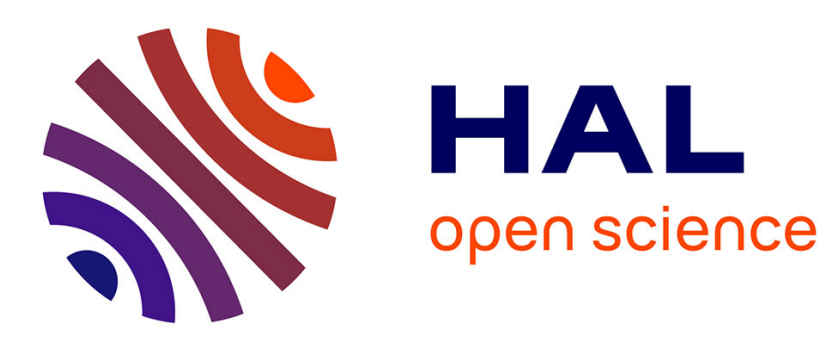

\title{
Theoretical Study of the Interaction of Electron Donor and Acceptor Molecules with Graphene \\ Tao Hu, I.C. Gerber
}

\section{To cite this version:}

Tao Hu, I.C. Gerber. Theoretical Study of the Interaction of Electron Donor and Acceptor Molecules with Graphene. Journal of Physical Chemistry C, 2013, 117 (5), pp.2411-2420. 10.1021/jp311584r . hal-02366365

\section{HAL Id: hal-02366365 https://hal.science/hal-02366365}

Submitted on 15 Nov 2019

HAL is a multi-disciplinary open access archive for the deposit and dissemination of scientific research documents, whether they are published or not. The documents may come from teaching and research institutions in France or abroad, or from public or private research centers.
L'archive ouverte pluridisciplinaire HAL, est destinée au dépôt et à la diffusion de documents scientifiques de niveau recherche, publiés ou non, émanant des établissements d'enseignement et de recherche français ou étrangers, des laboratoires publics ou privés. 


\title{
Theoretical Study of the Interaction of
}

\section{Electron Donor and Acceptor Molecules with}

\author{
Graphene
}

Tao Hu, Iann C. Gerber*

Université de Toulouse; INSA, UPS, CNRS; LPCNO

135 avenue de Rangueil, F-31077 Toulouse, France 
Abstract:

With the aim of understanding recent experimental data concerning non-covalent $n / p$ doping effects in grapheme samples, we have investigated the interactions between two prototypical donor and acceptor molecules and graphene mono and bi-layers systems, by means of density functional theory calculations. We report and rationalize the structural, thermodynamical aspects, as well as charge transfers and the induced electronic structure modifications of the graphenic substrates in interaction with tetrathiafulvalene (TTF), an organic donor molecule and tetracyanoethylene (TCNE), a typical acceptor. If the results show that $p$-doping of a graphene monolayer due to TCNE molecules, can occur even at low concentration, n-doping of graphene requires either larger concentrations or cooperative adsorption of TTF molecules. In both cases, non-covalent doping only implies shifts of the Fermi level, and keeps the linear dispersion of the $\pi$ and $\pi^{*}$ state around the Dirac point. Moreover the intercalation of donor/acceptor molecules decouples the layers and doped them.

Keywords: non-covalent functionalization, graphene, Charge Transfer, DFT 


\section{Introduction}

Graphene, a truly two dimensional monolayer of $s p^{2}$-bonded carbon, is a very inspiring material, since its unique physical properties, especially the ballistic electron transport, ${ }^{1}$ could lead to many applications. For instance, in the case of graphene based devices, a key issue is to tune the carrier concentration by shifting the Fermi level of the graphene above or below the Dirac point, where the density of states is zero. ${ }^{2}$ This can be realize either by applying electric field,,$^{3-5}$ with a gate voltage, or chemically. ${ }^{6,7}$

Chemical doping is an effective method to tailor the electrical properties of graphene, by controlling the hole or electron concentrations and at the same reducing unwanted contamination. A recent review ${ }^{8}$ has been devoted to present the chemical functionalization of graphene state of the art. One promising route, to achieve a fine tunable graphene device, is the use of non-covalent interaction. This solution offers two main advantages over covalent doping. Firstly it does not disturb the graphenic lattice, ${ }^{9,10}$ leaving the electronic structure intact. ${ }^{11}$ Secondly this process is completely reversible, contrary to covalent doping that randomly adds back-scattering centers.

Moreover the electronic behavior of experimental samples of graphene is totally dependent on the growth methods. ${ }^{12}$ In some cases few layers graphene (FLG) can be obtained. It is well documented that the stacking geometries impact the electronic behavior of graphene, and one can imagine that intercalation process should control the doping in the case of FLG samples.

Several electron donor and electron acceptor (EDA) molecules have been extensively used for non-covalent doping of graphene, or single walled carbon 
nanotubes (SWNTs), see Ref [13] for a recent review. Substantial experimental studies have been performed to modify the properties of carbon nanostructures and understand changes in their electronic structures. Several experimental works have investigated interactions between carbon nanostructures and EDA molecules, such as TCNQ, ${ }^{14,15}$ TTF, ${ }^{16,17}$ TCNE, ${ }^{18,19}$ and F4-TCNQ. ${ }^{20}$ Charge transfer induced by EDA molecules is usually indirectly observed, but $n$-doping and $p$-doping have been reported mainly by Raman spectroscopy. However the amount of charge transfer is seldom reported due to the difficulty of quantifying the charge transfer by a proper measure in any experimental studies. To obtain a direct estimate of charge transfers and a better understanding of their effects, theoretical studies are mandatory. For instance, studies based on density functional theory (DFT), on TCNQ,${ }^{21}$ benzene, ${ }^{22,23}$ DDQ, ${ }^{11,22} \mathrm{TTF}^{21,22,24}$ or TCNE ${ }^{10,25}$ molecules adsorbed on graphene or SWNTs have been reported. In substance, their main conclusions are that non-covalent functionalization definitively leads to electronic structure's modifications. For instance F4-TCNQ, TCNQ and TCNE molecules can pull electrons from graphene, while TTF or $\mathrm{TDAE}^{10}$ molecules can give electrons to graphene. Usually this $\mathrm{CT}$ is determined by a Mulliken population analysis.

One has to keep in mind that the description of ground state CT complex using standard DFT calculations is still challenging. It is well known that Local Density Approximation (LDA) of the exchange-correlation functional are known to overestimate the binding energies of strong CT complexes, ${ }^{26}$ and the CT values. Exchange-correlation functionals based on Generalized Gradient Approximations (GGA) usually perform better, ${ }^{27}$ and improved results can be obtained with, first the inclusion of an amount of exact exchange and more importantly with the addition of a dispersion-term that account for weak interactions. 
In this study, we have investigated theoretically the binding, the CT of a typical acceptor molecule (TCNE) and a prototypical donor (TTF) with graphene monolayer and bilayer. We have studied the effects of the inclusion of van der Waals forces, by a non-empirical correction. Weak interactions definitively stabilize the system, yielding larger binding energies, but more importantly provide more reliable geometries. For graphene ML, an efficient $p$-doping is obtained, around 0.5 e for a molecular concentration of one percent approximately, in the case of a withdrawing molecule, without a strong change in the electronic structure. Larger concentrations of adsorbate are needed to obtain similar CT values in the case of $n$-doping by TTF. In the case of bilayer, intercalation of EDA molecules decouples the two MLs and efficiently dopes the system. The paper is organized as follow: next section is devoted to the computational settings and model presentations. Then results dealing with graphene monolayer and bilayers are reported in section 3, followed by the conclusion.

\section{Computational details}

We have performed total-energy DFT calculations with the Vienna ab initio simulation package VASP..$^{28-31}$ The code uses the full-potential projector augmented wave (PAW) framework. ${ }^{32,33}$ A $400 \mathrm{eV}$ cutoff energy for the plane-wave basis set was used. In order to compare our results with previous ones, we have used the semi-local Perdew-Burke-Ernzerhof (PBE) ${ }^{34}$ and $\mathrm{LDA}^{35}$ exchange-correlation (xc)-functionals. We have also used the vdW-DF functional, ${ }^{36,37}$ that accounts for dispersion effects self-consistently, in conjunction with PBE xc-functional for short-range effects. We are aware of recent results dealing about accuracy of vdW-DF scheme ${ }^{38}$ and the crucial choice of the xc-functional to describe short-range effects. ${ }^{39-41}$ Using this 
scheme, we were able to obtain a correct interlayer distance in graphite $3.4 \AA$, despite a value of the cleavage energy (65 meV/atom) larger than a more accurate estimate ${ }^{42}$ (56 meV/atom). All atoms have been fully relaxed until the forces on individual atoms were smaller than $0.02 \mathrm{eV} \AA^{-1}$. The Brillouin zones (BZ) of the calculation cells have been sampled according to a $\boldsymbol{\Gamma}$-centered grid. Besides, $k$-points sampling has been determined by converging total energy within several meV.

Considering the models to describe the molecule/graphene at several adsorption's situations and at various concentrations, we have considered: 1 molecule on a graphene ML made of 98 carbon atoms (7x7 primitive cells), 1 molecule inserted in a bilayer (98 carbon atoms for each layer), 2 molecules adsorbed on both sides of one ML with 98 carbon atoms, 1 molecule on graphene ML made of 24 carbon atoms (3x4 primitive cells) and 2 molecules on the same ML. Additionally we have carefully chosen a sufficient vacuum length in the $(\mathrm{O} z)$ direction to avoid spurious interaction between periodic images.

We have employed Bader Charge Analysis method ${ }^{43}$ to estimate the charge transfer in the different xc-functional approximations, using Bader program of $\mathrm{G}$. Henkelman's group. ${ }^{44-46}$ In order to follow the tracks of electronic structure changes of the molecules, numerical derivatives were used to calculate harmonic frequencies of the adsorbed molecules and compared to freestanding molecule situations.

To discuss thermodynamically the stability of the various configurations, the key parameter is the adsorption energy. It is defined for a molecule on a carboneous substrate as:

$$
E_{a d s}=\left(E_{(\text {mol } / \text { graph })}-E_{(\text {graph })}-n E_{(\text {mol })}\right) / n
$$


where $\mathrm{n}$ stands for the number of adsorbed molecules. One needs to subtract the total energy of the carbon nanostructure $\left(\mathrm{E}_{\text {(graph) }}\right)$ and the freestanding molecule energy $\left(\mathrm{E}_{(\mathrm{mol})}\right)$ from the total energy $\left(\mathrm{E}_{(\mathrm{mol} / \mathrm{graph})}\right)$ of the relaxed molecule on the carbon-based system.

According to the highly symmetrical geometries of TTF, TCNE molecules and the honeycomb structure of the carboneous substrates, we have considered four different configurations, as in a previous theoretical work. ${ }^{25}$ The corresponding orientations of the molecules are presented in Figure 1. Additionally we have tested a non-symmetrical configuration (Figure 1-B(c)), denoted displaced position in the following. The configuration's name refers to the relative position of the central $\mathrm{C}=\mathrm{C}$ bond of the adsorbed molecule with respect to the graphenic plan.

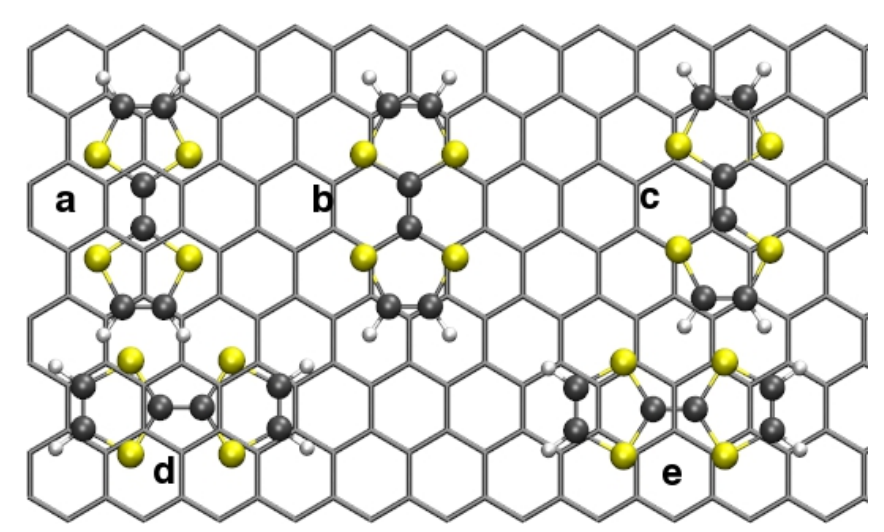

A

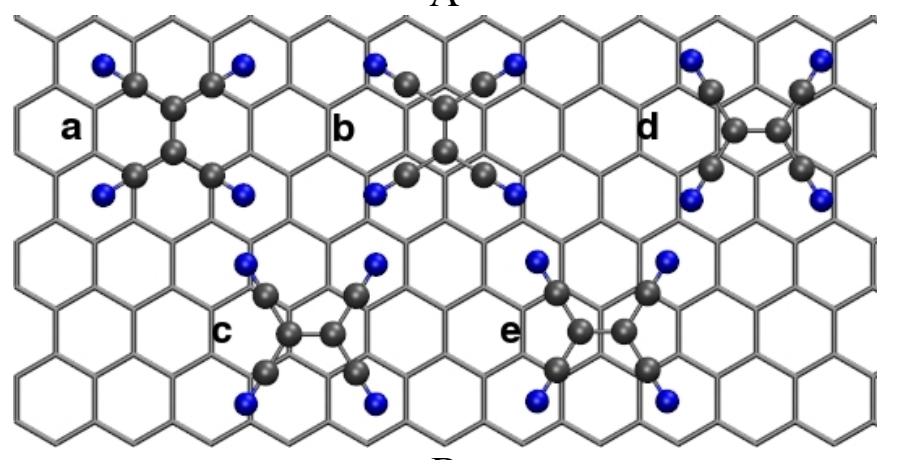

B

Figure 1. Top view of schematic structures of different adsorption configurations for TTF on graphene panel A: a. Hollow-I, b. Bridge-I, c. Displaced, d. Hollow-T, e. Bridge-T. TCNE on graphene panel B: a. Bridge-I, b. Hollow-I, c. Displaced, d. Hollow-T, e. Bridge-T. The grey lines represent the flat graphene layer. The carbon atoms are in grey, nitrogen atoms are in blue, hydrogen atoms are in white, and yellow balls stand for sulfur atoms. 


\section{Results and Discussion}

\subsection{Adsorption modes of TTF@graphene}

The Hollow-I, Hollow-T, Bridge-I, Bridge-T and the displaced adsorption modes of a single TTF molecule on graphene are depicted in Figure 1-A. Some geometry changes due to the interaction between TTF and graphene are observed, mainly at the molecule level with the three tested xc-functionals. The molecule is bent, with a dihedral angle of $162^{\circ}$ defined by the $\mathrm{C}=\mathrm{C}$ central bond and two hydrogen atoms at the molecule extremity), while the graphene ML stays almost flat. Although the molecule is curved, its geometric key parameters like bond-length vary only little (within $0.04 \AA$ ), while angles retain the values of the freestanding TTF molecule. The equilibrium distance between the TTF molecule and the graphene layer, defined as the distance between the middle $\mathrm{C}=\mathrm{C}$ bond and the graphene layer depends on the xc-functional choice. As expected, the larger value is for PBE (3.6 ̊), while vdW-DF reduces it by $0.1 \AA$. The LDA equilibrium distance is $3.4 \AA$, which can be viewed as a first indication of a too strong delocalization of the molecular electronic density.

Table 1(a) summarizes adsorption energies of the different configurations for TTF/graphene system in PBE, LDA, and vdW-DF respectively. The most stable configuration is the "Bridge-T" configuration (Figure 1-A(e)) for the three tested functionals. This result is consistent with previous studies. ${ }^{22}$ Indeed using LDA, Sun et $a l^{24}$ report a value of $-16.6 \mathrm{kcal} / \mathrm{mol}$ for the adsorption energy in LDA, while in Ref [21], the adsorption energy is about $-7 \mathrm{kcal} / \mathrm{mol}$ for the same adsorption mode. Mind that these differences can be attributed to various computational settings and cell calculations. 
TABLE 1: Adsorption Energy (in $\mathrm{kcal} / \mathrm{mol}$ ) of TTF(a) and TCNE(b) on graphene Monolayer and charge transfer (in elementary charge unit (e)) between molecules and graphene in the case of five different sites, for 3 different flavors of exchangecorrelation functionals, at low concentration (1 molecule per $98 \mathrm{C}$ atoms).

a)

\begin{tabular}{ccccccc}
\hline \multirow{2}{*}{ Configuration } & \multicolumn{2}{c}{ (PBE) } & \multicolumn{2}{c}{ (LDA) } & \multicolumn{2}{c}{ (vdW-DF) } \\
\cline { 2 - 7 } & $\mathrm{E}_{\text {ads }}$ & $\mathrm{CT}$ & $\mathrm{E}_{\mathrm{ads}}$ & $\mathrm{CT}$ & $\mathrm{E}_{\mathrm{ads}}$ & $\mathrm{CT}$ \\
\hline Hollow-I & -1.5 & 0.11 & -12.8 & 0.21 & -28.7 & 0.06 \\
Bridge-I & -1.5 & 0.12 & -12.0 & 0.19 & -28.6 & 0.07 \\
Displaced & -1.6 & 0.13 & -13.0 & 0.21 & -28.6 & 0.07 \\
Hollow-T & -1.5 & 0.12 & -13.2 & 0.20 & -28.7 & 0.06 \\
Bridge-T & -1.7 & 0.11 & -14.1 & 0.20 & -29.2 & 0.07
\end{tabular}

b)

\begin{tabular}{ccccccc}
\hline \multirow{2}{*}{ Configuration } & \multicolumn{2}{c}{ (PBE) } & \multicolumn{2}{c}{ (LDA) } & \multicolumn{2}{c}{ (vdW-DF) } \\
\cline { 2 - 7 } & $\mathrm{E}_{\mathrm{ads}}$ & $\mathrm{CT}$ & $\mathrm{E}_{\mathrm{ads}}$ & $\mathrm{CT}$ & $\mathrm{E}_{\text {ads }}$ & $\mathrm{CT}$ \\
\hline Bridge-I & -8.4 & -0.45 & -17.8 & -0.44 & -31.8 & -0.45 \\
Hollow-I & -9.3 & -0.44 & -19.5 & -0.44 & -32.9 & -0.46 \\
Displaced & -8.4 & -0.44 & -18.8 & -0.44 & -32.4 & -0.46 \\
Hollow-T & -9.1 & -0.46 & -19.4 & -0.45 & -32.7 & -0.49 \\
Bridge-T & -8.3 & -0.47 & -17.9 & -0.46 & -31.9 & -0.48
\end{tabular}

Interestingly the adsorption energies of five sites are almost equal for one particular choice of functional. It reveals that the TTF molecule can move or even rotate relatively easily, especially at room temperature, at very low energetic cost. When comparing the three tested functionals, LDA binds the system with an adsorption energy of around $-13 \mathrm{kcal} / \mathrm{mol}$, while very weak binding is yielded with PBE. By including non-local correlation terms, i.e. with the vdW-DF functional, a stronger binding is obtained, more than twice LDA $E_{\text {ads }}$ values, as a result of van der Waals forces contribution. It means that, as explained in the recent study of Steinmann et al, ${ }^{27}$ dispersion forces have a significant additive stabilization's effect on CT systems. An indirect proof of the existence of this weak interaction, between the two subsystems, comes from the comparison of specific frequencies of the TTF 
molecule before and after adsorption. A tiny shift of the central $\mathrm{C}=\mathrm{C}$ bond frequency is observed from $1525 \mathrm{~cm}^{-1}$ to $1489 \mathrm{~cm}^{-1}$ with PBE functional. This clearly indicates that there is only weak binding between a graphene ML and TTF. It is mainly a physisorption-dominant process only, which does not alter the electronic structure of the molecule, as observed experimentally. ${ }^{14}$ PBE provides no binding at all, by missing non-local correlations and by not compensating them by a cancellation of errors as in the LDA case.

The Table 1(a) also presents CT between the two subsystems, after charge integration over atomic basins as defined by a Bader analysis. Independently of the adsorption modes, a small charge transfer from the molecule to the graphene layer is estimated both in PBE and vdW-DF. The CT value is roughly of $0.1 \mathrm{e}$ for a concentration of round $1 \%$, since there is one molecule adsorbed per $98 \mathrm{C}$ atoms. On the contrary, a charge transfer significantly larger $(0.2 \mathrm{e})$ occurs with LDA. These values are in good agreement with previous studies that report a CT of $0.1 \mathrm{e}^{24}$ and $0.26 \mathrm{e}^{22}$ for LDA. To understand this discrepancy between LDA and GGA results, one has to remind that, as reported in a recent theoretical work, ${ }^{47}$ LDA intrinsically tends to delocalize the electron density, while GGA usually contract it. This CT overestimate appears as a pure artifact of the xc-functional used, and results of a larger overlap between the "too-delocalized" electronic densities of the two subsystems. Indeed, since a work of J. Harris ${ }^{48}$ in the 80 's, it is known that, at small overlaps, the balance in the total energy, between the exchange term and the kinetic term, is broken in the LDA scheme contrary to a Hartree-Fock calculation. This provides a spurious binding from the exchange contribution of the Hamiltonian, with an equilibrium distance usually too small. Besides, one has to note that the effect of 
the non-local correlation on CT values is very small, and this process is governed by exchange mainly.

A last evidence of the very weak effect of the TTF molecule adsorption is obtained by comparing the Band Structure (BS) of TTF/graphene system with the pristine graphene situation obtained at the PBE level. As it is shown in Figure 2(b), the presence of the well-localized molecular state placed just in the vicinity of the Fermi level, and a very tiny gap opening are the only changes in the BS induced by TTF adsorption, at this particular concentration. Contrarily to previous theoretical studies, TTF at low concentration does not $n$-dope graphene. Since the physisorption of a TTF molecule does not disturb the electronic structure of graphene, it means that the interaction of this particular electron donor stays weak as soon as the concentration is around $1 \%$.

(a)

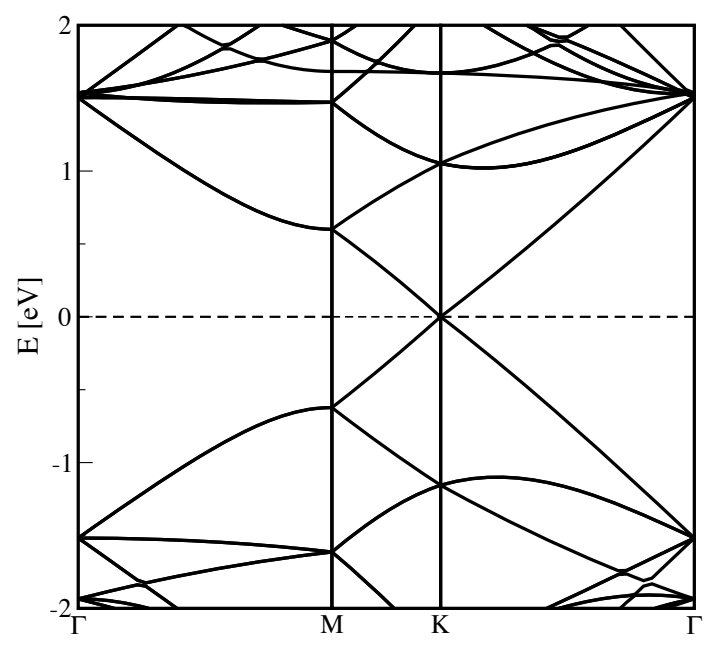


(b)

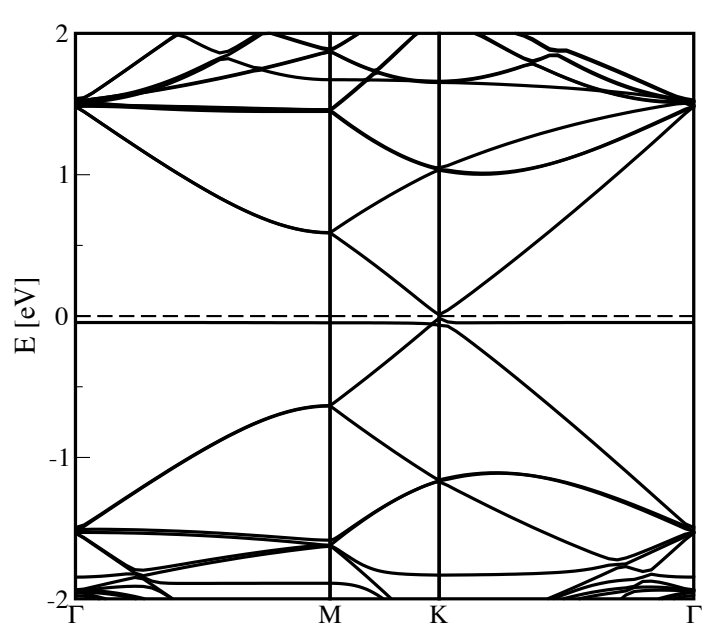

(c)

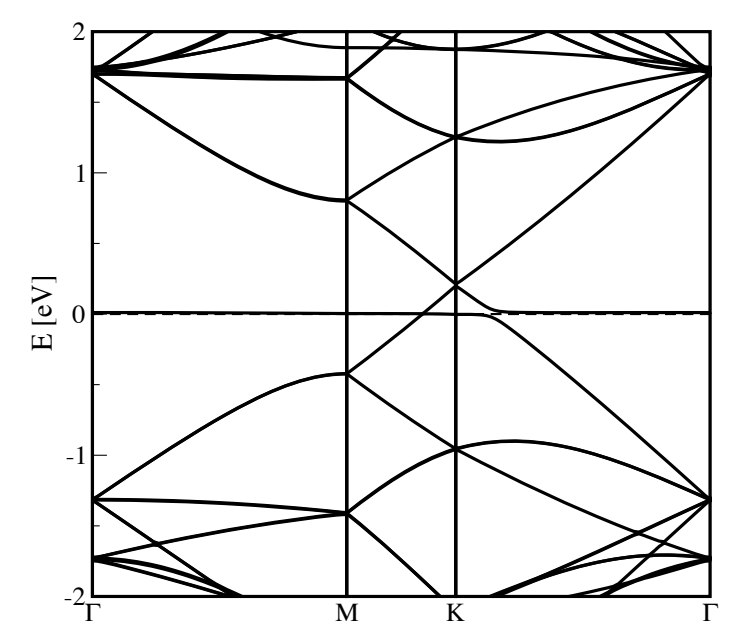

Figure 2: Band structures of pristine graphene (a), TTF/graphene system (b), and $\mathrm{TCNE} /$ graphene system (c) at low concentration.

\subsection{Adsorption modes of TCNE@graphene}

Figure (1-B) depicts the five tested configurations of the TCNE@graphene system at low concentration. After relaxation, the TCNE molecule stays flat and parallel to the graphene layer. Distance between TCNE and the honeycomb layer is about 3.0, 3.2, and $3.3 \AA$ in LDA, vdW-DF and PBE respectively. A first indication of a CT occurring between the two subsystems, is the significant change in the bond- 
length of the central $\mathrm{C}=\mathrm{C}$ from 1.38 to $1.41 \AA$, without inducing strong deformations of the graphenic network.

The adsorption energy in Table 1(b) provides interesting information. TCNE is bound to graphene, no matter the choice of the xc-functional, contrarily to the prototypical donor molecule, i.e. TTF. The most stable configuration is the "HollowI" site shown in Figure (1-B(b)), for the three tested xc-functionals. This result is in good agreement with previous theoretical works ${ }^{10,25}$ for LDA and PBE. The inclusion of long-range correlations stabilizes by almost $24 \mathrm{kcal} / \mathrm{mol}$ the system when compared to the PBE value. This large value results probably of our choice to use $\mathrm{PBE}+\mathrm{vdW}-\mathrm{DF}$ functional, and we agree that another choice of short-range exchange functional would certainly reduce this absolute value. Besides, similar to the TTF's case, TCNE also has good mobility on graphene, due to small energy differences among the different high-symmetry adsorption sites. One should also notice that at this low concentration of adsorbates, TTF and TCNE have very similar adsorption energy values on graphene ML.

When looking at the frequency of the central $\mathrm{C}=\mathrm{C}$ bound, at the PBE level, we have found a significant downshift from 1516 to $1413 \mathrm{~cm}^{-1}$, which is compatible with the observed increase of the bond-length. This can be viewed as a first evidence of a change in the molecular electronic structure that may originate from a partial CT from the graphene to the molecule. Indeed, direct estimate of the CT from graphene to TCNE is detected. The corresponding values for the three functionals are presented in Table 1(b). In all cases, a charge of $\sim 0.46 \mathrm{e}$ is transferred from graphene to TCNE at this particular low concentration. The agreement with previous estimates $0.3 \mathrm{e},{ }^{10} 0.44$ $\mathrm{e}^{25}$ and with reported values with parent molecules like TCNQ $(0.3 \mathrm{e})^{10}$ and F4-TCNQ $0.3 \mathrm{e},{ }^{49} 0.4 \mathrm{e},{ }^{24} 0.5 \mathrm{e}^{10}$ is clear. Locally, the charge value of each individual atom 
indicates that this loss of charge in graphene is attributed to the carbon atoms in the close vicinity of the adsorbed TCNE. On the other side, the charge gained by TCNE mostly settles on the $\mathrm{N}$ atoms of cyano groups and, to a lesser extent, on the two central $\mathrm{C}$ atoms.

This CT has a drastic consequence in the electronic structure of the graphenic substrate. The band structure (BS) of the TCNE/graphene system is presented in Figure 2(c). The main feature is the alignment of the occupied molecular state and the Fermi level of the total system. As a consequence the Fermi level is shifted below the typical K-point crossing of the pristine graphene by a value of $0.2 \mathrm{eV}$, leaving unchanged the linear dispersion around this point. Thanks to this pure $p$-type doping the substrate becomes metallic. This interesting property is in good agreement with a significant drop in the resistivity of graphene sample doped by TCNE. ${ }^{18}$ As a consequence at the same concentration, $p$-doping is much more efficient that $n$-doping of graphene, as it has been proposed based on experimental evidences. ${ }^{17,19,50}$ Additionally a non-crossing point just after point $\mathrm{K}$ appears in the BS at the Fermi Level, meaning that the occupied molecular charged state has the same symmetry as the $\pi$ states of graphene.

The following subsection is devoted to rationalize CT mechanism with graphene ML thanks to the adaptation of the Integer Charge Transfer (ICT) model widely in use in the context of energy-alignment at organic/metal interface. ${ }^{51}$

\subsection{Charge Transfer mechanism of graphene}

Recently based on the conventional Newns-Anderson model, ${ }^{52}$ Nistor et al ${ }^{53}$ have proposed that graphene acts as a metallic surface to understand the chemical 
disproportionation of adsorbed dopant layers into CT complexes. Basically, the key parameter that should control the $p$-doping process is the energy difference between the lowest unoccupied molecular orbital (LUMO) energy and the work function of the graphene. In the same spirit, to rationalize the $n$-doping mechanism, simply by mirroring the electron and hole density, the key parameter that control the CT, should be the energy difference between the Ionization Potential (IP) of the molecule, i.e the highest occupied molecular orbital (HOMO), and the work function of the graphene. In the ICT model, $, 5,55$ which describes, with success, interfaces that are characterized by a negligible hybridization of molecular orbitals and substrate states, CT occurs through tunneling, since the substrate work function is greater than the formation energy of a positively charge donor state ( $\mathrm{D}^{+}$in the following) for $n$-doping or since the substrate work function is smaller than the formation energy of a negatively charge acceptor state $\left(\mathrm{A}^{-}\right.$in the following) for $p$-doping. These molecular charged states $\mathrm{D}^{+} / \mathrm{A}^{-}$can be viewed as derived-states of HOMO or LUMO ones, lying in the molecular band-gap. They take into account for the changes in electronic and geometrical structures of the adsorbed species after release/withdrawing of electronic density, and interestingly they also depend on the substrate's screening meaning that the distance between the adsorbate and the graphenic substrate is a key parameter.

Figure 3 summarizes the $p$-doping process that corresponds to the TCNE/Graphene situation. As proposed by Braun et al, ${ }^{51}$ one can represent the CT mechanism in three steps. Figure 3 panel 1, shows the energetic configuration before bringing together the two subsystems. Again, we recall that to yield a significant CT, the work function $\Phi_{\mathrm{Gr}}$ has to be smaller than $E_{A^{-}}$, the energy of the negatively charged state of the molecule. By reducing the distance (Fig. 3 panel 2) electronic density starts to flow from the substrate to the molecule, creating a dipole at the interface, due 
to the positively charged substrate facing a negatively charged molecule. This dipole increases the work function of the hybrid system, in other words, it downshifts the Fermi level, leaving the BS of the substrate unchanged. This increase of the work function continues until the alignment between $E_{F}$ and $E_{A^{-}}$is reached, Figure 3 panel 3. The resulting work function $\Phi_{\mathrm{A}^{-} / \mathrm{Gr}}$ is equal to $E_{A^{-}}$, meaning that the Fermi level is pinned to the molecular negatively charged state at the interface. $\Delta$ the energy corresponding to the interface dipole is equal to the difference between the energy $E_{A^{-}}$ and $\Phi_{\mathrm{Gr}}$. 


\section{$\Phi_{\mathrm{Gr}}<\mathrm{E}_{\mathrm{A}^{-}}$}
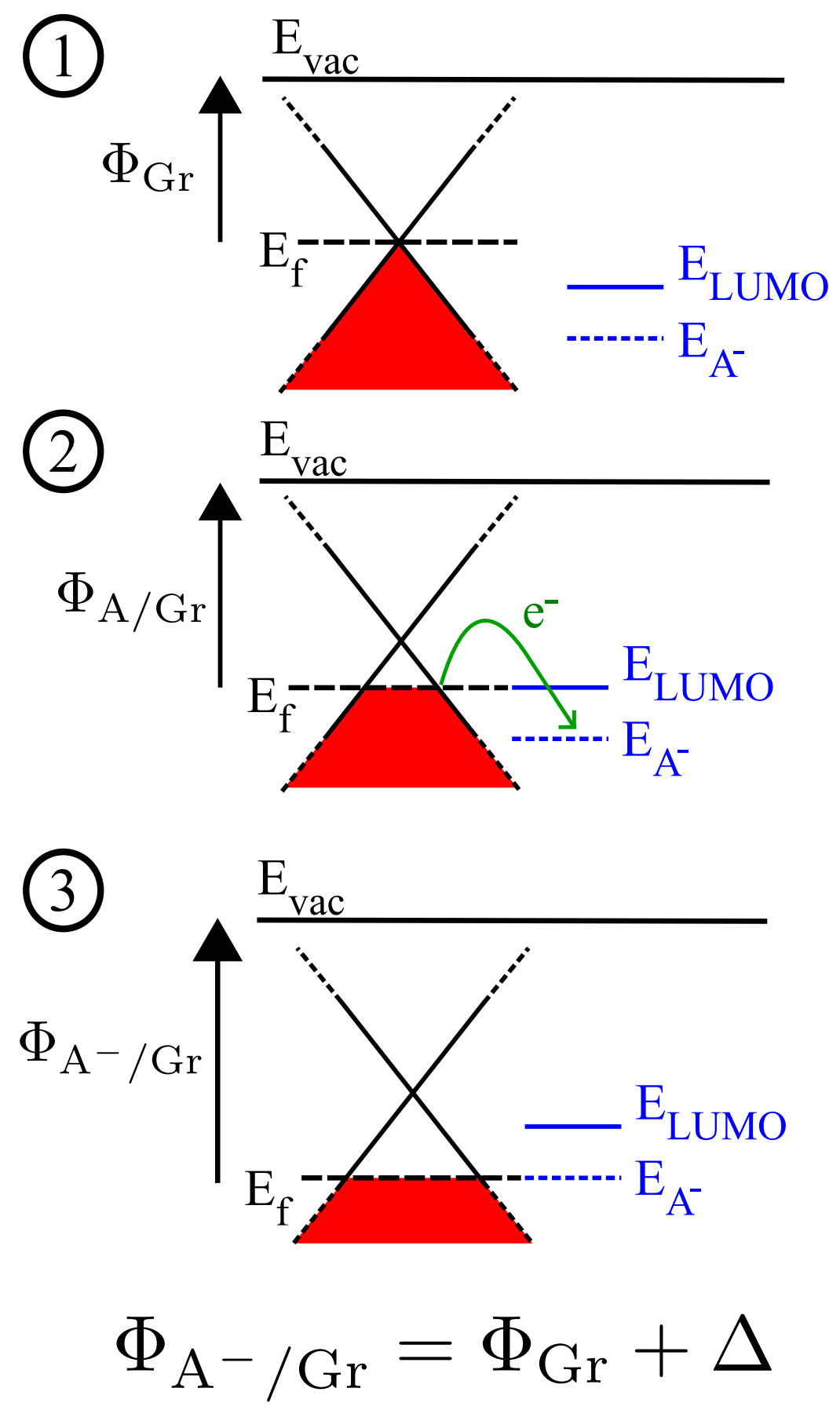

Figure 3: Scheme of $p$-doping process: panel 1 presents the energy levels before the interaction starts. Panel 2 shows intermediate situation, with the electronic flow, while Panel 3 corresponds to the final energy alignment. 


\section{$\Phi_{\mathrm{Gr}}>\mathrm{E}_{\mathrm{D}^{+}}$}
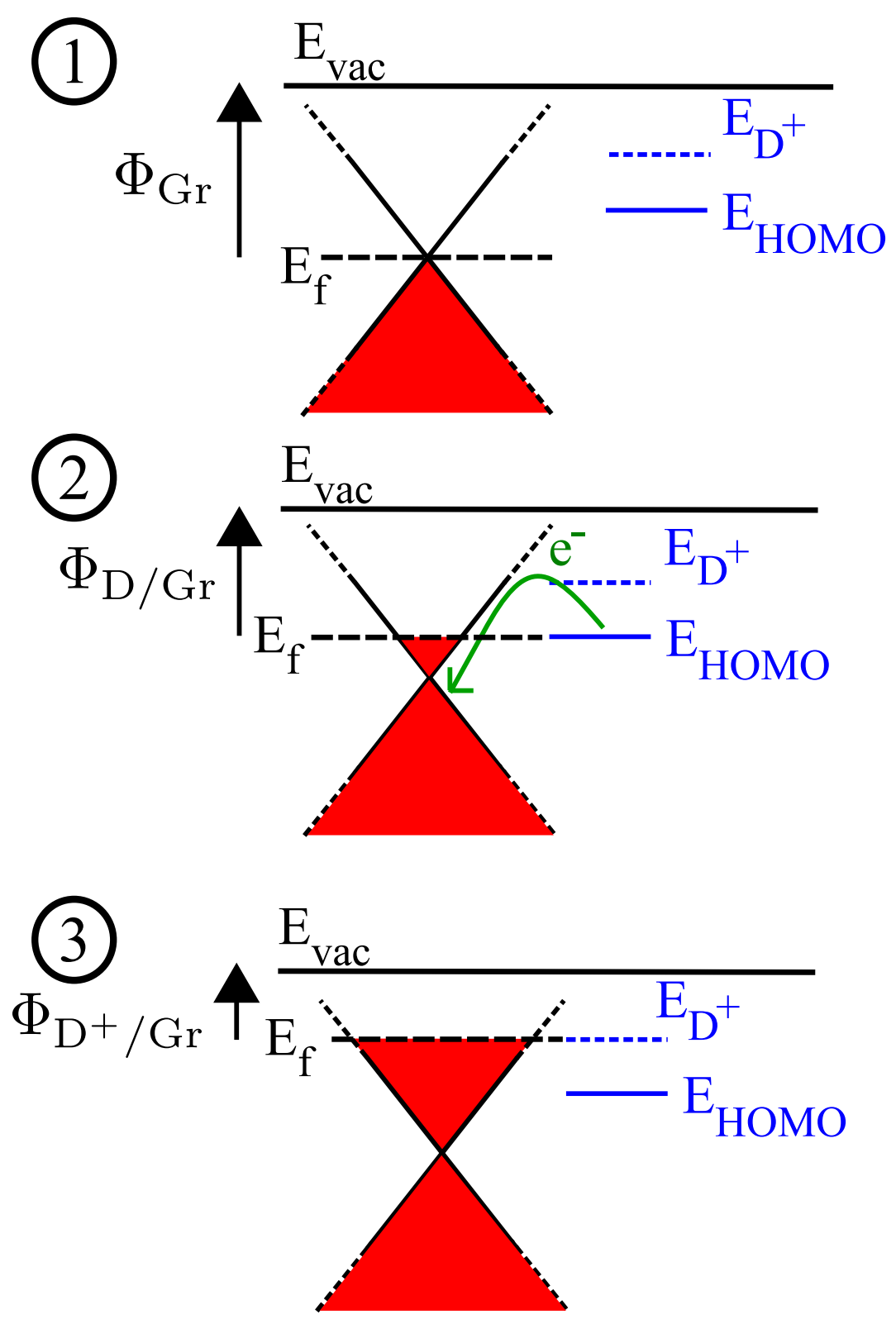

$$
\Phi_{\mathrm{D}^{+} / \mathrm{Gr}}=\Phi_{\mathrm{Gr}}-\Delta^{\prime}
$$

Figure 4: Scheme of $n$-doping process: panel 1 presents the energy levels before the interaction starts. Panel 2 shows intermediate situation, with the electronic flow, while Panel 3 corresponds to the final energy alignment.

Figure 4 shows a schematic view of the $n$-doping process as one could expect to happen in a typical donor/graphene interaction case. The initial requirement is that 
the work function $\Phi_{\mathrm{Gr}}$ has to be greater than $E_{D^{+}}$, the energy of the positively charged state of the molecule. Bringing the donor molecule in contact with the graphene ML, (Fig. 4 panel 2) electronic density start to leave the molecule to fill empty states of the substrate. In return it creates a dipole that originates from the negatively charged substrate facing a positively charged molecule. This dipole now decreases the work function of the hybrid system, that shifts up the Fermi level. The equilibrium is obtained when $E_{F}$ matches $E_{D^{+}}$. The resulting work function $\Phi_{\mathrm{D}+/ \mathrm{Gr}}$ is equal to $E_{D^{+}}$, meaning that the Fermi level is pinned to the molecular positive charged state at the interface. The work function difference before and after adsorption represents the energy corresponding to the created dipole at the interface. No CT will occur if $\Phi_{\mathrm{Gr}}$ is smaller than $E_{D+}$, or $\Phi_{\mathrm{Gr}}$ is larger than $E_{A^{-}}$. A so-called vacuum level alignment holds for this type of interface.

This model helps us to understand non-covalent $p$-doping of a graphene ML by TCNE molecules. Indeed by comparing the work function of the graphene sheet before $\left(\Phi_{\mathrm{Gr}}=4.25 \mathrm{eV}\right)$ and after adsorption $\left(\Phi_{\mathrm{A}^{-} / \mathrm{Gr}}=4.85 \mathrm{eV}\right)$, we have another indirect evidence of CT. This increase of the work function is in good agreement with experimental determinations, usually with an upshift of around $0.3-0.5 \mathrm{eV}$ as reported in Ref. [51] in the context of organic semi-conductor/conducting substrate interfaces.

Theoretically speaking, the situation is not ideal as well. Indeed the fact that semi-local xc-functional usually underestimates molecular band-gaps, ${ }^{56}$ starts to be problematic in order to predict quantitative results. For instance, in the TCNE case, we estimate that the LUMO energy of TCNE is $5.87 \mathrm{eV}$, a value largely overestimate when compared to the experimental one. Additionally our DFT estimate of the work function $(4.2 \mathrm{eV})$ is slightly smaller than the value proposed in a previous theoretical report $^{57}$ and the recent experimental determination. ${ }^{58}$ The overestimate of the LUMO 
energy combined with the underestimate of the work function, in standard DFT calculations, provides the necessary condition, by fortuitous cancellation of errors, that yields CT between TCNE and the graphene ML.

After adsorption of a single TTF molecule on the $(7 \times 7)$ primitive cell model of graphene, no significant change in the work function of the hybrid system is observed, meaning that with semi-local xc-functional calculations, we obtain a vacuum-level alignment regime at the interface only. The well-reported underestimate of the HOMO energy in standard DFT calculations is present here too, with a value of 4.7 $\mathrm{eV}$. Besides, by extrapolating the $E_{D^{+}}$value, with a minoring of $0.5 \mathrm{eV}$ as it is observed experimentally, ${ }^{51}$ the negatively charged state still lies below the Fermi level of the graphene sheet. This provides explanation for the absence of CT between TTF and our graphene ML at low concentration.

Experimentally speaking if one compares brute data, i.e the ionization potential of the TTF molecule $(6.70 \mathrm{eV}),{ }^{59}$ and the work function of graphene (4.6 $\mathrm{eV}$ ), and even if one estimates roughly the $\mathrm{D}^{+}$state energy by using the same percentage of reduction as in some reported experimental data, see Ref [51] and references therein, no CT should be observed experimentally. As far as the electronic states of the molecules do not mix with surface states of the substrate, an image charge contribution is present. ${ }^{60}$ In the case of metallic substrates, one can expect a significant reduction of the energy of the $\mathrm{D}^{+}$state, following this law $1 /\left(4 * \mathrm{R}_{\mathrm{ads}}\right)$ with the distance of the adsorbate to the surface. Hence, the positively charged state can be significantly higher in the molecular HOMO-LUMO gap. We have checked this assumption in a series of calculations, in which we have reduced the distance between the TTF molecule and the graphene sheet, from the equilibrium distance. As reported in Figure 5, one can see that CT increases when the distance gets smaller, which is an 
indirect proof of this image charge effect observed experimentally. As it is reported in the next subsection, increasing the concentration of adsorbates can compact the structure, i.e reducing the average distance to the graphenic substrate.

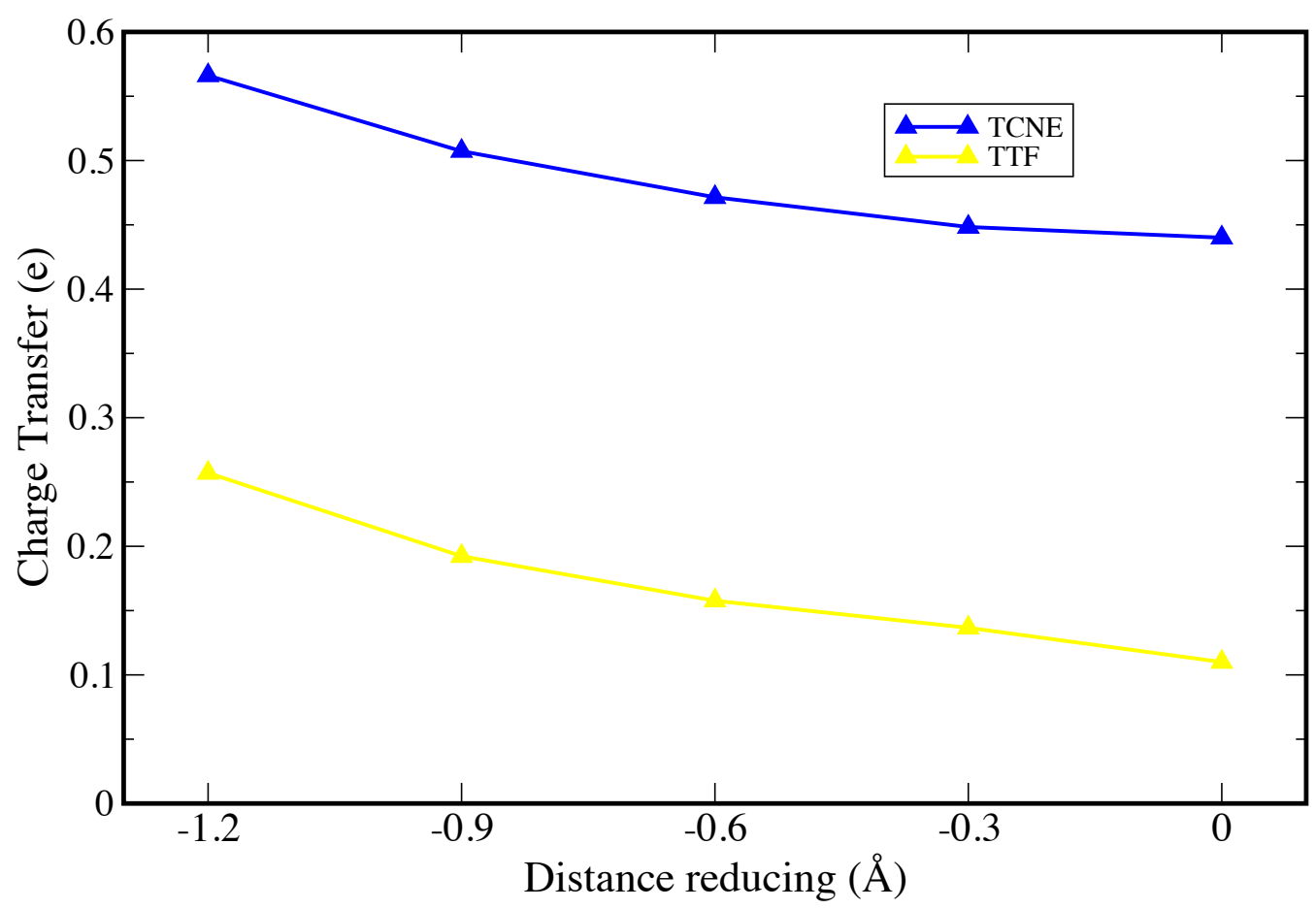

Figure 5: Charge transfer (in elementary charge unit (e)) versus the distance reducing from the equilibrium distance of TTF and TCNE on graphene, at low concentration. It means that distance reducing 0 stands for equilibrium position while $-1.2 \AA$ stands for a decrease of $1.2 \AA$ from the equilibrium distance.

Clearly, tuning the work function of graphene could be essential to the use of graphene for transparent electrodes, see reference [61] for instance, since Fermi level of graphene stays well aligned with the hole-injecting molecules, avoiding Ohmic contact or Schottky barrier. Varying CT by changing adsorption modes, concentration, could be of first importance for possible applications; this is discussed in the next paragraph. 


\subsection{Tuning the CT mechanism of graphene}

To study the influence of the concentration of the adsorbed molecules the practical way is to reduce the calculation's cell: we have chosen to test a situation of one molecule per $24 \mathrm{C}$ atoms. At this particular coverage value, the intermolecular distance becomes relatively small: $2.4 \AA$ for TTF and $2.6 \AA$ for TCNE and has strong structural consequences. Firstly this leads to an enhancement of the lateral intermolecular interaction, but a weakening of the interaction between molecules and the graphene layer. This is reflected in the change of the molecule/graphene distances enlarged by $0.4 \AA$ and $0.3 \AA$ for TTF and TCNE respectively. Secondly the small intermolecular distances cause intermolecular repulsion that distorts molecular structures drastically. For example, TTF becomes more curved, the dihedral angle is now $149^{\circ}$; the angle of $\mathrm{C} \equiv \mathrm{N}-\mathrm{C}=\mathrm{C}$ changes from 118 to 115 degrees to reduce repulsion from steric effect. Interestingly bond lengths of adsorbed molecule remain almost identical as in the low concentration situation. As expected, the adsorption energy is less important than at low concentration, in other word the systems are less bound $(7.4 \mathrm{kcal} / \mathrm{mol})$ due to lateral interactions. This may partially explain experimental results, in which donor molecules tend to bind less to graphene than acceptor molecules. ${ }^{14}$

Considering the CT at this concentration, TTF molecule does not transfer density to the graphene layer. On the contrary a charge of $0.18 \mathrm{e}$ per TCNE molecule is transferred from graphene to TCNE. Although total charge transfer decreases in high concentration, charge transfer per carbon atom increases from 0.0046 (1 molecule per $98 \mathrm{C}$ atoms) to 0.0075 . This result is compatible with the evolution of 
the Raman upshift of the G-band with respect to the dopant concentration; see Ref $[18,19]$ for instance. Since the charge transfer remains mainly local (limited in space), the molecular packing over the substrate is a key parameter to enhance the charge transfer.

Additionally doubling the concentration, (two molecules on a 24-carbon layer), is about to explain a recently published experimental work. It has been reported that when an effective dopant like F4-TCNQ is densely packed on epitaxial graphene film, most of the molecules are standing up..$^{62}$ The authors have proposed that the dopant molecules would be perpendicular to the graphene layer, since the dopant concentration becomes significant. To verify this, we have studied two different configurations: two molecules perpendicular to graphene and two molecules parallel to graphene at large coverage value. The relaxation process starting from two molecules parallel to graphene shows that this configuration is highly unstable. Balance between intermolecular repelling, limiting space and attractive interaction with the graphene layer slantingly aligns the molecules on graphene. See Figure 6 panel (a) for geometry's snapshots, of this double molecular layer configuration. Energetically speaking, when 2 TTF molecules interact with the graphene ML, the slanting adsorption mode is more stable, by more than $10 \mathrm{kcal} / \mathrm{mol}$, (Table 2) than the perpendicular mode, due to the strong repelling between the molecules.
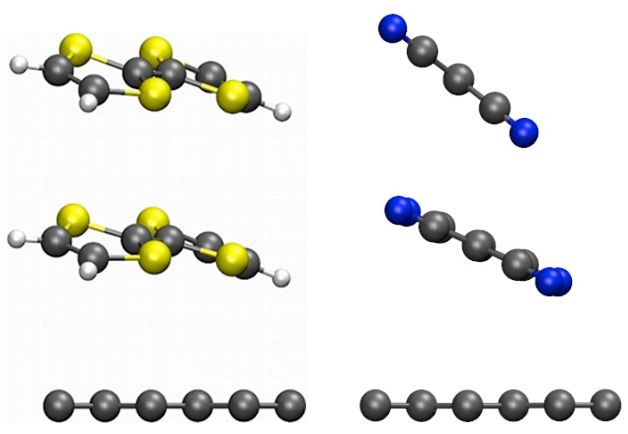
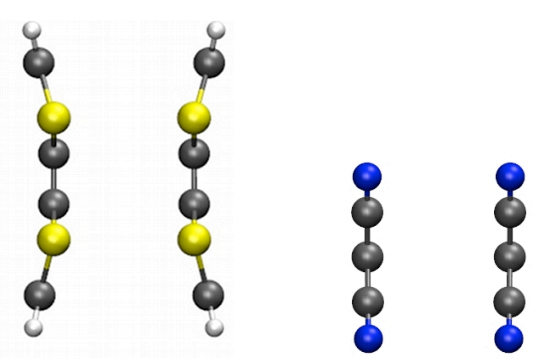
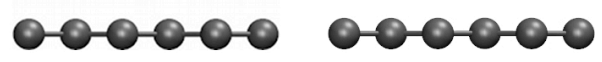
Figure 6: Side view of two molecules adsorbed on a graphene monolayer at high concentration, 2 molecules per $24 \mathrm{C}$ atoms. Panel a: Two TTF molecules (left) and two TCNE molecules (right) slantingly adsorbed on graphene ML. Panel b: Two TTF molecules (left) and two TCNE molecules (right) perpendicularly adsorbed on graphene ML.

TABLE 2: Adsorption Energy (in $\mathrm{kcal} / \mathrm{mol}$ ) of TTF and TCNE on Graphene Monolayer at a High Concentration (2 molecule per $24 \mathrm{C}$ atoms) and Charge Transfer (in elementary charge unit (e)) Between Molecules and Graphene.

\begin{tabular}{cccccc}
\hline & & \multicolumn{2}{c}{ PBE } & \multicolumn{2}{c}{ vdW-DF } \\
\cline { 3 - 6 } & & $\mathrm{E}_{\mathrm{ads}}$ & $\mathrm{CT}$ & $\mathrm{E}_{\mathrm{ads}}$ & $\mathrm{CT}$ \\
\hline \multirow{2}{*}{ TTF } & Slanting & -2.1 & 0.02 & -31.6 & 0.03 \\
& Perpendicular & -0.7 & 0.06 & -21.9 & 0.05 \\
\multirow{2}{*}{ TCNE } & Slanting & -2.1 & -0.11 & -20.7 & -0.11 \\
& Perpendicular & -2.8 & -0.17 & -21.2 & -0.18
\end{tabular}

In the case of acceptor molecules (like TCNE) the configuration with two molecules perpendicular to graphene (see Figure $6(\mathrm{~b})$ ) is stabilized by only 0.5 $\mathrm{kcal} / \mathrm{mol}$. This simply means that in the TCNE case, slanting and perpendicular adsorption modes are degenerate. This result confirms the proposed schematic deposition of F4-TCNQ molecules on graphene ML grown on SiC in Ref [62]. Indeed in both configurations two types of $\mathrm{N}$ atoms would be detected in XPS experiments, the ones closer to the graphene layer with a more pronounced $\mathrm{N}^{-1}$ character and the others staying uncharged. Moreover, the perpendicular configuration enhances the CT for the graphene to the TCNE molecules: doubling again the concentration leads to a doubling of the CT per carbon atom, from $0.0075 \mathrm{e} / \mathrm{C}$ to $0.015 \mathrm{e} / \mathrm{C}$. This phenomenon originates from the fact that in perpendicular configuration the two molecules interact with the graphene ML.

Another possible situation has been explored: the symmetrical and displaced co-adsorption of two molecules on both side of a graphene ML. The adsorption 
energy per molecule stays close to one obtained by a single adsorbed molecule: -29.2 $\mathrm{kcal} / \mathrm{mol}$ and $-30.7 \mathrm{kcal} / \mathrm{mol}$ for TTF and TCNE in symmetrical co-adsorption configuration respectively, while the adsorption energy for TTF and TCNE in displaced co-adsorption mode is -29.6 and $-31.1 \mathrm{kcal} / \mathrm{mol}$. The distance between molecule and graphene remains the same as in the one-side adsorption mode, in both cases. The graphene ML is slightly buckled in opposite direction due to displaced adsorption of molecules, however, it remains flat in symmetrical co-adsorption mode. The graphene ML gives $0.30 \mathrm{e}$ to each TCNE, and each TTF provides $0.09 \mathrm{e}$ to graphene layer. This adsorption mode also enhances CTs from the graphene point of view. This significantly modifies the electronic structure of the graphene ML, as it can be seen in Figure 7(a) and (b). For instance with the 2TTF/graphene system, the upshift of the Fermi level is now $0.36 \mathrm{eV}$, while it is not significant for one molecule adsorbed. With two TTF molecules symmetrically adsorbed, graphene is more effectively $n$-doped, probably due to the fact that the adsorption of a first molecule on one side decreases the work function of the hybrid ML compared to the pristine one and favors a CT on the other side, which in return reduces the work function. Meanwhile, for 2TCNE/graphene system, the Fermi level is shifted down by $0.57 \mathrm{eV}$ when comparing to the doped graphene by a single molecule case. To summarize, below a certain concentration (around 8\%), doping effect on graphene due to noncovalent interaction can be reinforced by increasing dopant's concentration, i.e with increasing the molecular packing. 
(a)

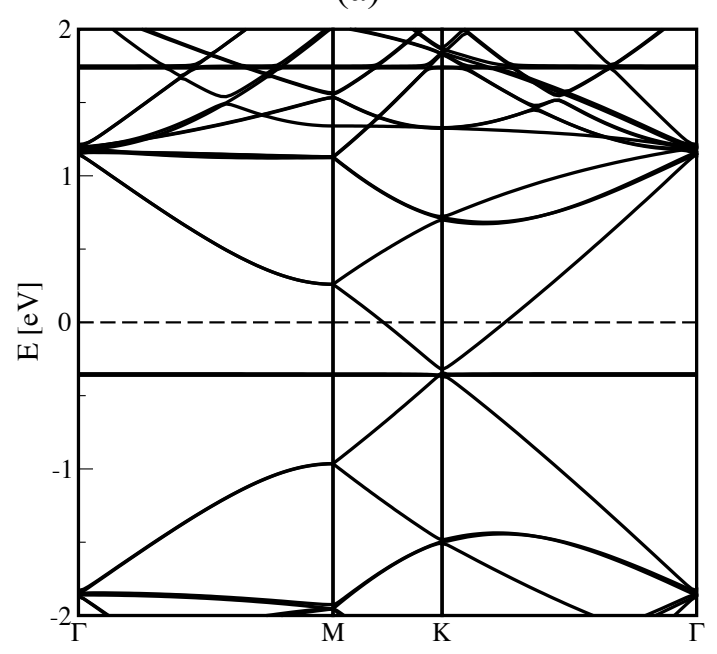

(b)

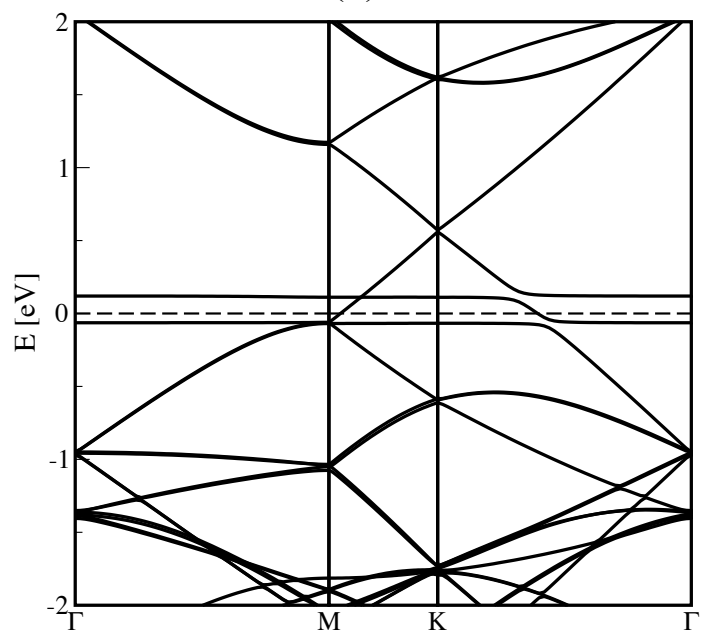

Figure 7: Band structures of symmetric TTF/graphene/TTF hybrid system (a) and TCNE/graphene/TCNE (b) at low concentration.

Without a good control in the synthesis process, graphene samples usually appear to be few layers graphene (FLG) instead of a single monolayer (ML). For instance the samples used in recent experiments ${ }^{17-19,50}$ to study by Raman spectroscopy CT effects on graphene, are typically made of 2 to 6 layers. To investigate the interaction with FLG, we have intercalated TTF and TCNE molecule 
between two graphene MLs presenting an AA-stacking and AB-stacking. In this situation the concentration of adsorbate is one molecule per $196 \mathrm{C}$ atoms. The interlayer spacing is known to be crucially dependent to the xc-functional choice. At the vdW-DF level, the AA-stacking layers are stabilized with an interlayer spacing of $6.4 \AA$ and $6.2 \AA$ for TTF and TCNE molecule respectively. Besides, the distance between the layers in the AB-stacking is $0.2 \AA$ smaller than in the AA-stacking situation. When the calculations are performed with the pure PBE functional, the separations between two layers are $6.8 \AA$ and $6.4 \AA$ for TTF and TCNE respectively, regardless of bilayer's stacking mode. In both cases, the molecule just locates in the middle of interlayer spacing, and remains completely flat due to symmetrical interactions. However, the two graphene MLs are buckled independently of the molecule; the two closest carbon atoms to the molecule moved in opposite directions of the intercalated molecule by almost $0.2 \AA$.

(a)

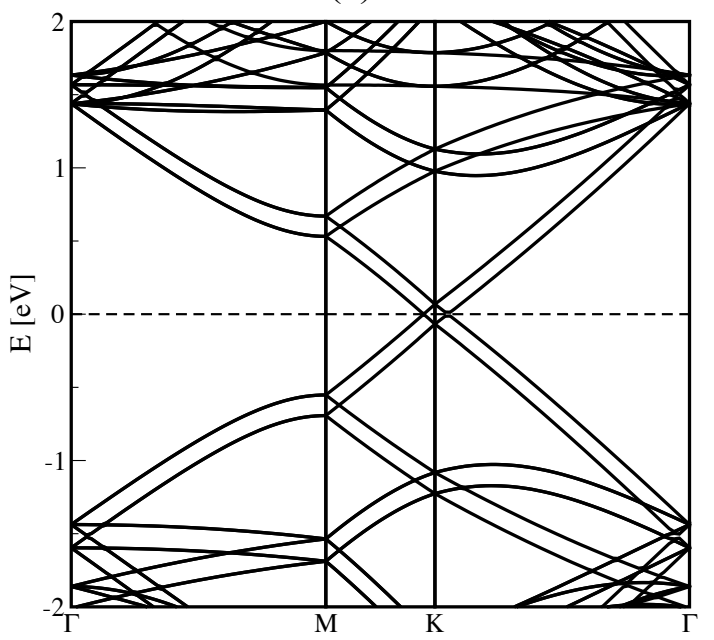

(c) (b)

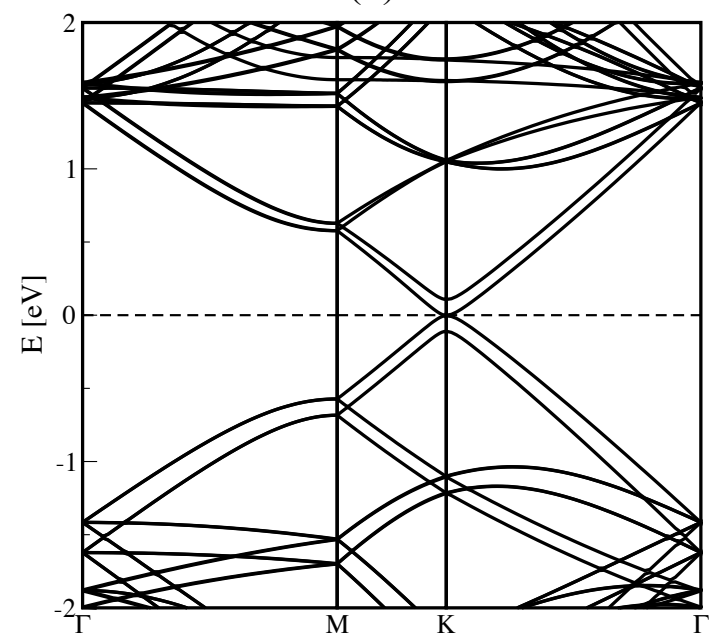

(d) 

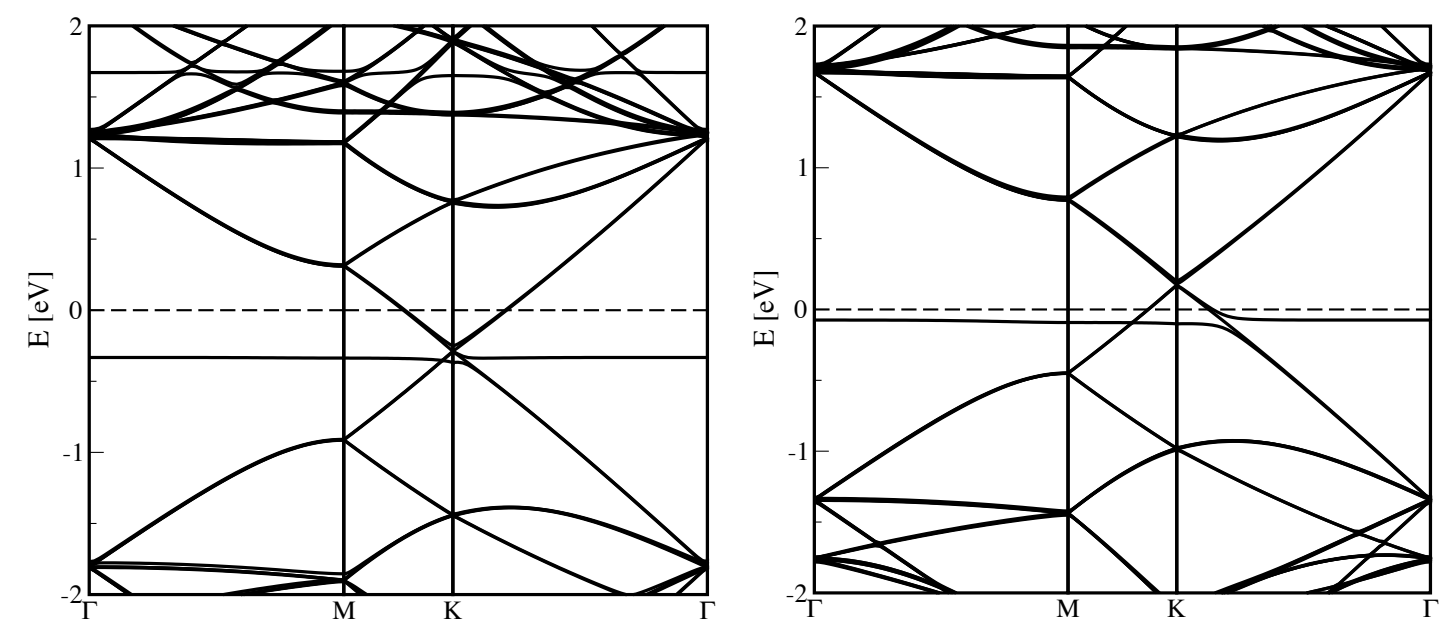

(e)

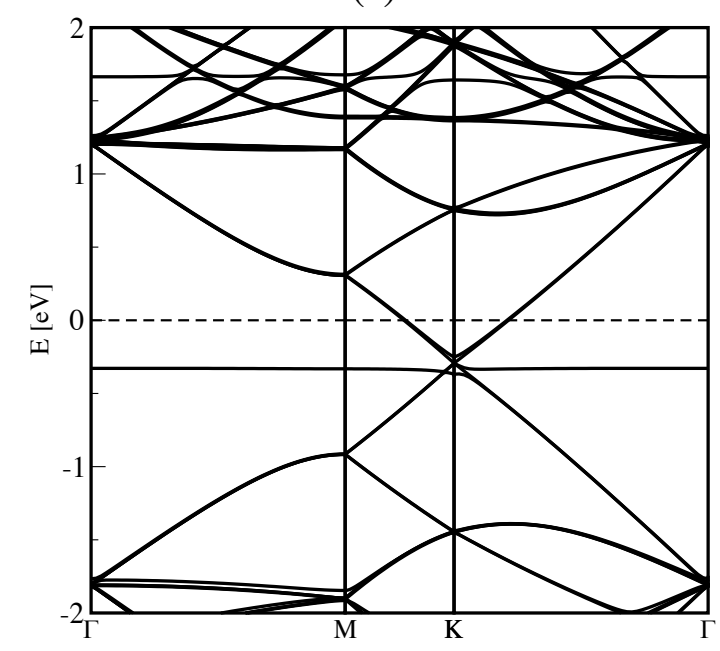

(f)

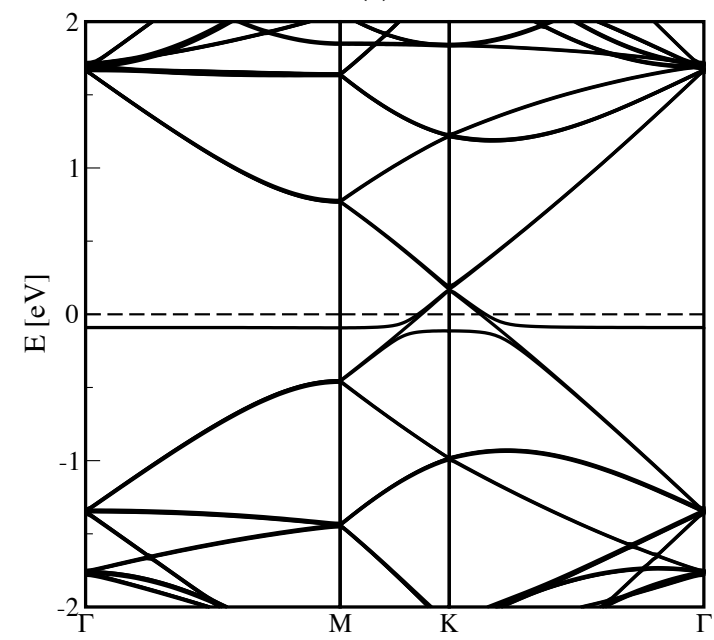

Figure 8: Band structures of pristine AA-stacking (a) and AB-stacking bilayer (b), TTF (c) and TCNE (d) intercalated into an AA-bilayer graphene, while (e) and (f) panels are for intercalated $\mathrm{AB}$-bilayer systems.

Interestingly, TTF looses 0.18 e to the two layers; meaning that for each layer, the charge transfer value is equal to the one observed in the monolayer case, for AAstacking, while the AB-bilayer obtains 0.19e from the TTF molecule. TCNE gets 0.61e from the AA-bilayer. Same value is yielded for the AB-stacking system. Each layer contributes less than in the isolated situation probably due to a saturation of the molecular electronic density. The effect of the doping is visible in the BSs shown in 
Figure 8(c) and (d). Energy bands at low energies near $\mathrm{K} / \mathrm{K}^{\prime}$ are still linear dispersed, which are different from electronic structure of a pair of coupled graphene MLs. ${ }^{63}$ This is due to the large interlayer distance that decouples the layers due to electrostatic effect, since each ML is now charged. Therefore, the intercalation of molecules into AA-stacking or AB-stacking makes almost no difference in the BS's. Indeed, the two possible bilayer have nearly the same binding energy ${ }^{64,65}$ and band structure $^{66}$ when the interlayer spacing is greater than $4 \AA$, when they decouple. A resulting downshift of $0.17 \mathrm{eV}$ for Fermi level is observed in the case of TCNE intercalation, an upshift of $0.29 \mathrm{eV}$ in the case of TTF.

\section{Conclusion}

Adsorption of the two electron donor/acceptor molecules does not cause significant structural distortion in the honeycomb carbon network of a graphene monolayer. Nevertheless slight buckling has been observed in the two-side adsorption mode or in the intercalation of TCNE or TTF molecules. Interestingly there is no evidence of chemical bond's formation between the two organic molecules and the graphenic nanostructures. It means that the interaction is physisorption mainly, which reveals the fundamental role played by van der Waals' forces. Indeed a good description of these weak interactions are mandatory in such hybrid systems, since to yield a correct charge transfer, a correct equilibrium distance is needed, due to charge image effect. Since standard semi-local functionals usually provide too large distances, one can expect an underestimation of the charge transfer. That's also why LDA which usually provides too small equilibrium distances, tends to overestimate CT. Non-covalent doping either by holes of electrons have been reported and 
rationalized by a simple model, in the case of graphene monolayer and bilayer. Basically, thanks to a simple model of CT through an organic/metal interface, based on derived HOMO or LUMO states, the capability of molecules to dope graphene ML.

We have shown that it is possible to control the carrier type and concentration in graphene by adjusting of dopant's type, concentration and adsorption modes. At low concentration, $p$-doping of graphene is already efficiently realized by TCNE molecules, while $n$-doping is one order in magnitude less important when a graphene ML interacts with TTF molecules. Mind that an increase of the doping is observed, by means of CT value increase, when the concentration is increased. This statement is in good agreement with experimental works, in which it is usually indirectly observed by shifts in Raman spectroscopy. In the case of TTF molecules, two-side adsorption mode provides better CT than one-side adsorption mode. Moreover intercalation of acceptor/donor molecules decouples the two layers but also dope them.

It could be interesting to study extensively the effect of curvature, with respect to the charge transfer, since the $s p^{3}$ character of $\mathrm{C}$ atoms is pronounced in small diameter nanotubes while one should recover graphene behavior for very large diameter SWNTs. Moreover, since work-functions of semi-conductor tubes are different from metallic ones up to a certain threshold value, ${ }^{67}$ one can expect variations of CT with different chiral indexes for tubes presenting small diameters. Works are in progress in this direction.

\section{Acknowledgments}


The authors would like to thank P. Puech and Pr. R. Poteau for fruitful discussions. They also thank the CALcul en MIdi-Pyrénées (CALMIP, grant 2011/2012-P0812) for generous allocations of computer time. Part of this work was also performed using HPC resources from GENCI-[CINES] (Grant 2011/2012-6357). Finally, T. Hu would like to thank CSC-INSA consortium for financial support for his PhD study. 


\section{References and Notes}

(1) Das Sarma, S.; Adam, S.; Hwang, E. H.; Rossi, E. Rev. Mod. Phys. 2011, 83, 407-470.

(2) Geim, A. K.; Novoselov, K. S. Nature Mater. 2007, 6, 183-191.

(3) Novoselov, K. S. Science 2004, 306, 666-669.

(4) Zhang, Y.; Tan, Y.-W.; Stormer, H. L.; Kim, P. Nature 2005, 438, 201-204.

(5) Novoselov, K. S.; Geim, A. K.; Morozov, S. V.; Jiang, D.; Katsnelson, M. I.; Grigorieva, I. V.; Dubonos, S. V.; Firsov, A. A. Nature 2005, 438, 197-200.

(6) Schedin, F.; Geim, A. K.; Morozov, S. V.; Hill, E. W.; Blake, P.; Katsnelson, M. I.; Novoselov, K. S. Nature Materials 2007, 6, 652-655.

(7) Wehling, T. O.; Novoselov, K. S.; Morozov, S. V.; Vdovin, E. E.; Katsnelson, M. I.; Geim, A. K.; Lichtenstein, A. I. Nano Lett. 2008, 8, 173-177.

(8) Georgakilas, V.; Otyepka, M.; Bourlinos, A. B.; Chandra, V.; Kim, N.; Kemp, K. C.; Hobza, P.; Zboril, R.; Kim, K. S. Chemical Reviews 2012, 112, 61566214.

(9) Pinto, H.; Jones, R.; Goss, J. P.; Briddon, P. R. Phys. Status Solidi (a) 2010, 207, 2131-2136.

(10) Chi, M.; Zhao, Y.-P. Comput. Mater. Sci. 2012, 56, 79-84.

(11) Tournus, F.; Latil, S.; Heggie, M. I.; Charlier, J.-C. Phys. Rev. B 2005, 72, 075431-075435.

(12) Park, S.; Ruoff, R. S. Nature Nanotechnology 2009, 4, 217-224.

(13) Rao, C. N. R.; Voggu, R. Materials Today 2010, 13, 34-40.

(14) Varghese, N.; Ghosh, A.; Voggu, R.; Ghosh, S.; Rao, C. N. R. J. Phys. Chem. C 2009, 113, 16855-16859.

(15) Yuge, R.; Yudasaka, M.; Maigne, A.; Tomonari, M.; Miyawaki, J.; Kubo, Y.; Imai, H.; Ichihashi, T.; Iijima, S. J. Phys. Chem. C 2008, 112, 5416-5422.

(16) Voggu, R.; Rout, C. S.; Franklin, A. D.; Fisher, T. S.; Rao, C. N. R. J. Phys. Chem. C 2008, 112, 13053-13056.

(17) Choudhury, D.; Das, B.; Sarma, D. D.; Rao, C. N. R. Chem. Phys. Lett. 2010, 497, 66-69.

(18) Voggu, R.; Das, B.; Rout, C. S.; Rao, C. N. R. J. Phys.: Condens. Matter 2008, 20, 472204-472208.

(19) Subrahmanyam, K. S.; Voggu, R.; Govindaraj, A.; Rao, C. N. R. Chem. Phys. Lett. 2009, 472, 96-98.

(20) Chen, W.; Chen, S.; Qi, D. C.; Gao, X. Y.; Wee, A. T. S. J. Am. Chem. Soc. 2007, 129, 10418-10422.

(21) Manna, A. K.; Pati, S. K. Chem. Asian J. 2009, 4, 855-860.

(22) Zhang, Y.-H.; Zhou, K.-G.; Xie, K.-F.; Zeng, J.; Zhang, H.-L.; Peng, Y. Nanotechnology 2010, 21, 065201-065207.

(23) Tournus, F.; Charlier, J.-C. Phys. Rev. B 2005, 71, 165421-165428.

(24) Sun, J. T.; Lu, Y. H.; Chen, W.; Feng, Y. P.; Wee, A. T. S. Phys. Rev. B 2010, $81,155403-155408$.

(25) Lu, Y. H.; Chen, W.; Feng, Y. P.; He, P. M. J. Phys. Chem. B 2009, 113, 2-5.

(26) Ruiz, E.; Salahub, D. R.; Vela, A. J. Am. Chem. Soc. 1995, 117, 1141-1142.

(27) Steinmann, S. N.; Piemontesi, C.; Delachat, A.; Corminboeuf, C. J. Chem. Theory Comput. 2012, 8, 1629-1640.

(28) Kresse, G.; Hafner, J. Phys. Rev. B 1993, 47, 558-561. 
(29) Kresse, G.; Hafner, J. Phys. Rev. B 1994, 49, 14251-14269.

(30) Kresse, G.; Furthmüller, J. Computational Materials Science 1996, 6, 15-50.

(31) Kresse, G.; Furthmüller, J. Phys. Rev. B 1996, 54, 11169-11186.

(32) Blöchl, P. E. Phys. Rev. B 1994, 50, 17953-17979.

(33) Kresse, G.; Joubert, D. Phys. Rev. B 1999, 59, 1758-1775.

(34) Perdew, J. P.; Burke, K.; Ernzerhof, M. Phys. Rev. Lett. 1996, 77, 3865-3868.

(35) Kohn, W.; Sham, L. J. Phys. Rev. 1965, 140, A1133-A1138.

(36) Andersson, Y.; Langreth, D. C.; Lundqvist, B. I. Phys. Rev. Lett. 1996, 76, 102-105.

(37) Gulans, A.; Puska, M. J.; Nieminen, R. M. Phys. Rev. B 2009, 79, 201105201108.

(38) Vydrov, O. A.; Van Voorhis, T. J. Chem. Phys. 2009, 130, 104105-104111.

(39) Klimeš, J.; Bowler, D. R.; Michaelides, A. Phys. Rev. B 2011, 83, 195131195143.

(40) Cooper, V. R. Phys. Rev. B 2010, 81, 161104-161107.

(41) Klimeš, J.; Bowler, D. R.; Michaelides, A. J. Phys.: Condens. Matter 2010, 22, 022201-022201-5.

(42) Spanu, L.; Sorella, S.; Galli, G. Phys. Rev. Lett. 2009, 103, 196401-196404.

(43) Bader, R. F. W. Atoms in Molecules - A Quantum Theory; Oxford University Press: UK, 1990.

(44) Henkelman, G.; Arnaldsson, A.; Jónsson, H. Comput. Mater. Sci. 2006, 36, 354-360.

(45) Sanville, E.; Kenny, S. D.; Smith, R.; Henkelman, G. J. Comput. Chem. 2007, 28, 899-908.

(46) Tang, W.; Sanville, E.; Henkelman, G. J. Phys.: Condens. Matter 2009, 21, 084204-084204-7.

(47) Mori-Sánchez, P.; Cohen, A. J.; Yang, W. Phys. Rev. Lett. 2008, 100, 146401-146404.

(48) Harris, J. Phys. Rev. B 1985, 31, 1770-1779.

(49) Pinto, H.; Jones, R.; Goss, J. P.; Briddon, P. R. J. Phys.: Condens. Matter 2009, 21, 402001-402001-3.

(50) Gopalakrishnan, K.; Moses, K.; Dubey, P.; Rao, C. N. R. J. Mol. Struct 2012, 1023, 2-6.

(51) Braun, S.; Salaneck, W. R.; Fahlman, M. Adv. Mater. 2009, 21, 1450-1472.

(52) NEWNS, D. M. Phys. Rev. 1969, 178, 1123-1135.

(53) Nistor, R. A.; Newns, D. M.; Martyna, G. J. ACS Nano 2011, 5, 3096-3103.

(54) Fahlman, M.; Crispin, A.; Crispin, X.; Henze, S. K. M.; Jong, M. P. de; Osikowicz, W.; Tengstedt, C.; Salaneck, W. R. Journal of Physics: Condensed Matter 2007, 19, 183202-183202-20.

(55) Tengstedt, C.; Osikowicz, W.; Salaneck, W. R.; Parker, I. D.; Hsu, C.-H.; Fahlman, M. Appl. Phys. Lett. 2006, 88, 053502-053504.

(56) Jones, R. O.; Gunnarsson, O. Rev. Mod. Phys. 1989, 61, 689-746.

(57) Leung, T. C.; Kao, C. L.; Su, W. S.; Feng, Y. J.; Chan, C. T. Phys. Rev. B 2003, 68, 195408-195413.

(58) Yan, R.; Zhang, Q.; Li, W.; Calizo, I.; Shen, T.; Richter, C. A.; Hight-Walker, A. R.; Liang, X.; Seabaugh, A.; Jena, D.; Grace Xing, H.; Gundlach, D. J.; Nguyen, N. V. Appl. Phys. Lett. 2012, 101, 022105-022105-4.

(59) Lichtenberger, D. L.; Johnston, R. L.; Hinkelmann, K.; Suzuki, T.; Wudl, F. Journal of the American Chemical Society 1990, 112, 3302-3307. 
(60) Zhao, J.; Pontius, N.; Winkelmann, A.; Sametoglu, V.; Kubo, A.; Borisov, A. G.; Sánchez-Portal, D.; Silkin, V. M.; Chulkov, E. V.; Echenique, P. M.; Petek, H. Phys. Rev. B 2008, 78, 085419-085425.

(61) Bae, G.; Jung, H.; Park, N.; Park, J.; Hong, S.; Park, W. Appl. Phys. Lett. 2012, 100, 183102-183105.

(62) Coletti, C.; Riedl, C.; Lee, D. S.; Krauss, B.; Patthey, L.; von Klitzing, K.; Smet, J. H.; Starke, U. Phys. Rev. B 2010, 81, 235401-235408.

(63) Mak, K. F.; Sfeir, M. Y.; Misewich, J. A.; Heinz, T. F. Proceedings of the National Academy of Sciences 2010, 107, 14999-15004.

(64) Nanda, B. R. K.; Satpathy, S. Phys. Rev. B 2009, 80, 165430-165436.

(65) Kolmogorov, A. N.; Crespi, V. H. Phys. Rev. B 2005, 71, 235415-235420.

(66) Okada, S.; Kobayashi, T. Jpn. J. Appl. Phys. 2009, 48, 050207.

(67) Su, W. S.; Leung, T. C.; Chan, C. T. Phys. Rev. B 2007, 76, 235413-235420. 

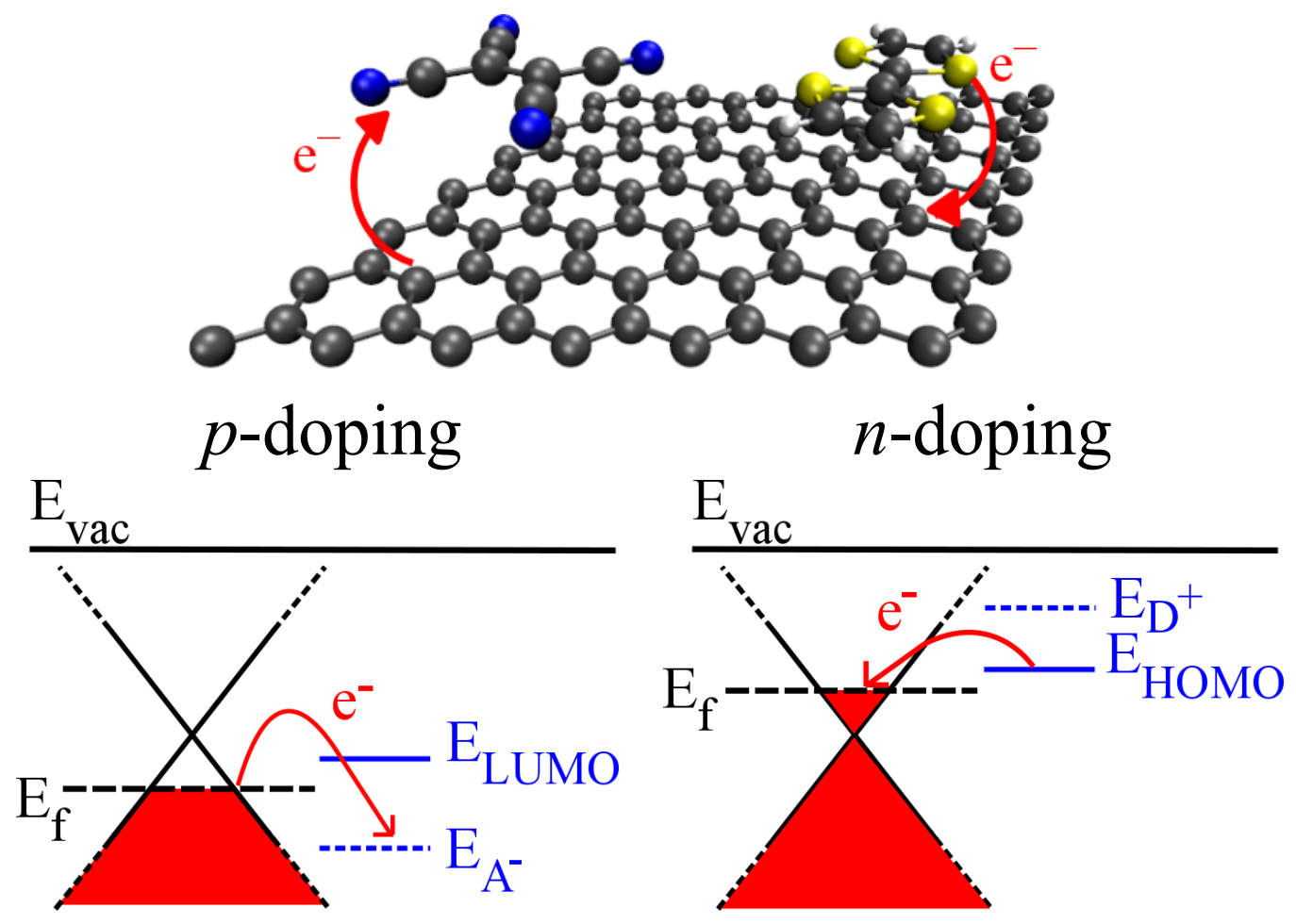